\title{
Standard quantum limits for broadband position measurement
}

\author{
Hideo Mabuchi \\ Norman Bridge Laboratory of Physics 12-33, California Institute of Technology, Pasadena, California 91125
}

(Received 28 January 1998)

\begin{abstract}
I utilize the Caves-Milburn model for continuous position measurements to formulate a broadband version of the standard quantum limit (SQL) for monitoring the position of a free mass and illustrate the use of Kalman filtering to recover the SQL for estimating a weak classical force that acts on a quantum-mechanical test particle under continuous observation. These derivations are intended to clarify the interpretation of SQL's in the context of broadband quantum measurement, with particular attention paid to the question of how it might be possible to verify that a given laboratory measurement does indeed achieve backaction-limited sensitivity. The method used to analyze force detection may be extended to the case of weak classical forces with arbitrary time dependence, including nonstationary and impulsive signals. [S1050-2947(98)06907-8]

PACS number(s): 03.65.Bz, 06.20.Dk, 42.50.Lc
\end{abstract}

Substantial efforts have been devoted to elaborating standard quantum limits (SQL's) for both discrete [1-4] and continuous $[4,5]$ measurements of the position of a free mass. In a large part, the motivation for such investigations stems from a pressing need to identify any possible constraints imposed by the principles of quantum measurement on the experimental possibility to detect gravitational waves $[6,7]$. My objectives in this paper will be to formulate a SQL appropriate to broadband continuous measurements of the position of a free mass (expressed in terms of the signal bandwidth and with measurement sensitivity given in units of length per $\sqrt{\mathrm{Hz}}$ ), to discuss its proper interpretation, and to demonstrate its compatibility with the usual SQL [4] for detecting a weak classical force. The mathematical analysis will be adapted to the continuous measurement model of Caves and Milburn [8], which appears to have direct relevance for concrete experimental scenarios such as atomic force microscopy [9] and cavity QED [10,11].

Although the limits I derive will be familiar from previous studies of the discrete and narrow-band measurement scenarios, my emphasis here will be on formulating these limits in a manner that is specific to broadband quantum measurement. In particular, I have found that some care needs to be taken in deriving a SQL for detecting weak forces from the SQL for continuous position measurement. The method I use below emulates the classical technique of Kalman filtering [12,13] and was motivated by the general strategy of quantum system identification discussed in [14]. Throughout this paper I have adopted the time-domain, statespace perspective of quantum measurement whose virtues have become apparent from recent advances in adaptive quantum measurement [15] and quantum feedback [16]. Such methods hold great promise for the challenge of formulating experimentally tenable strategies for broadband quantum-nondemolition measurement.

The mathematical formalism necessary to treat continuous quantum measurements has been developed by numerous authors, with the most relevant works for the present discussion being $[8,13,17]$. While it is not absolutely essential to go to the continuous limit, doing so will allow us to use the convenient notation of stochastic differential equations (SDE's) [18]. What really matters for the discussion at hand is an assumption that the time scale associated with measurement interactions and readouts is much shorter than any time scale on which we wish to understand the system dynamics.

In the Caves-Milburn model for continuous quantummechanical measurements of position [8] there is one system of interest and an infinite succession of identical (and identically prepared) "meters." Let the meters be labeled by an index $r$. The system is brought into momentary contact with the $r$ th meter at time $t_{r}=r \tau$ and the position operator of each meter is measured sharply just after it has interacted with the system. The string of measurement results thus generated constitutes a classical record of the system evolution. Continuous measurement is achieved in the limit where the timeinterval between measurements $\tau$ goes to zero, with the uncertainty of each individual measurement simultaneously going to infinity in an appropriate manner.

Let $x, p$ denote the system position and momentum operators and $X_{r}, P_{r}$ the position and momentum operators of the $r$ th meter. We adopt the following time-dependent interaction Hamiltonian between the system and the meters:

$$
H_{i n t}=\sum_{r=1}^{n} \delta(t-r \tau) x P_{r} .
$$

The delta-function form is chosen to facilitate the limit $\tau$ $\rightarrow 0$, but as discussed above it may be viewed as the idealization of any "shrinkable" function with compact support. We assume that each meter, just before it interacts with the system, is prepared in the pure state $\left|\Upsilon_{r}\right\rangle$ with the Gaussian wave function $\left(X_{r}\left|\xi_{r}\right\rangle \equiv \xi_{r}\left|\xi_{r}\right\rangle\right)$

$$
\left\langle\xi_{r} \mid \Upsilon_{r}\right\rangle \equiv \Upsilon\left(\xi_{r}\right)=\frac{1}{(\pi \sigma)^{1 / 4}} \exp \left[\frac{-\xi_{r}^{2}}{2 \sigma}\right]
$$

Caves and Milburn have derived an exact expression for the conditional evolution of the system state under the following measurement protocol: at each time $t_{r}$, (i) couple the system to the $r$ th meter prepared in state (2), (ii) evolve the system and meter under the interaction Hamiltonian (1), and (iii) perform a precise measurement of $X_{r}$. If we write $\rho\left(t_{r}-\right)$ 
for the system state just before the measurement at time $t_{r}$ $\equiv r \tau$, the postmeasurement system state $\rho\left(t_{r}+\right)$ is given by

$$
\begin{gathered}
\rho\left(t_{r}+\right)=\hat{\Upsilon}\left(\xi_{r}\right) \rho\left(t_{r}-\right) \hat{\Upsilon}^{\dagger}\left(\xi_{r}\right), \\
\hat{\Upsilon}\left(\xi_{r}\right) \equiv\left\langle\xi_{r}\left|e^{-i x P_{r} / \hbar}\right| \Upsilon_{r}\right\rangle,
\end{gathered}
$$

where $\xi_{r}$ corresponds to the actual outcome of the meter projection in stage (iii). Recall that $x, X_{r}, P_{r}$ are Hermitian operators, $\xi_{r}$ is a $c$ number, and $\left|Y_{r}\right\rangle$ is a Hilbert-space ket. $\hat{\Upsilon}_{r}$ is thus a quantum operation density. Qualitatively speaking, the basic effect of Eq. (3) is to shift the centroid $x_{r}^{\prime}$ $\equiv \operatorname{Tr}\left[x \rho\left(t_{r}-\right)\right]$ of the system's position-space distribution towards the value conveyed by $\xi_{r}$ and to reduce its overall width $\Delta_{r}^{\prime} \equiv \operatorname{Tr}\left[x^{2} \rho\left(t_{r}-\right)\right]-\left(x_{r}^{\prime}\right)^{2}$ by an amount that depends on the ratio $\Delta_{r}^{\prime} / \sigma$. Note that this is not a projective measurement: The postmeasurement state $\rho\left(t_{r}+\right)$ depends on both the measurement result $\xi_{r}$ and the premeasurement state $\rho\left(t_{r}-\right)$.

The operation (4) maps Gaussian pure states of the system to Gaussian pure states, so we can in fact parametrize the selective evolution of a free particle (initially prepared in a Gaussian pure state) by just four real numbers. Choosing $x_{r}$ $\equiv\langle x\rangle, \quad p_{r} \equiv\langle p\rangle, \quad \Delta_{r} \equiv 2\left\langle(\Delta x)^{2}\right\rangle, \quad$ and $\quad \varepsilon_{r} \equiv\langle\Delta x \Delta p$ $-\Delta p \Delta x\rangle / \hbar$, Caves and Milburn derived a set of difference equations for the evolution of these quantities with each successive measurement. They further showed that Gaussian initial states generally converge toward stationary evolutions, in which $x_{r}$ and $p_{r}$ evolve stochastically but $\Delta_{r}$ and $\varepsilon_{r}$ vary periodically: During the time intervals between measurements both widths increase according to the free Hamiltonian, but the effect of each measurement is to reduce them by a constant "contraction factor', $C$ such that $\Delta_{r}$ $\equiv \operatorname{Tr}\left[x^{2} \rho\left(t_{r}+\right)\right]-\left(x_{r}\right)^{2}$ is independent of $r$. The form of the evolution equations is especially convenient in this stationary regime, so in what follows we shall assume that stationarity has been bootstrapped by preparing the system in an appropriate initial state.

In order to derive a broadband SQL for position measurements, we start with the stationary difference equations (3.30a) and (3.30b) from [8]:

$$
\begin{gathered}
x_{r+1}-x_{r}=p_{r} \frac{\tau}{m}+\frac{C-1}{C}\left(\xi_{r}-x_{r}^{\prime}\right), \\
p_{r+1}-p_{r}=\frac{\hbar}{\sigma \sqrt{C}}\left(\xi_{r}-x_{r}^{\prime}\right) .
\end{gathered}
$$

Here unprimed quantities $x_{r}, p_{r}$ describe the system state just after the $(r-1)$ th measurement has been made and $x_{r}^{\prime}$ holds just before the $r$ th measurement.

We first need to rewrite the difference Eqs. (5) as a SDE by taking $\tau \rightarrow 0$ together with $\sigma \rightarrow \infty$ such that their product $D \equiv \sigma \tau$ stays constant. Recall, from Eqs. (3.20) and (3.21) of [8], that $\xi_{r}-x_{r}^{\prime}$ is a Gaussian random variable with zero mean and variance $\sigma C / 2$. In the continuous limit it also turns out that $C \rightarrow 1$ [8], so we immediately have an explicit (Ito) stochastic differential equation

$$
d\left(\begin{array}{l}
x \\
p
\end{array}\right)=\left(\begin{array}{c}
p / m \\
0
\end{array}\right) d t+\left(\begin{array}{c}
0 \\
\sqrt{\frac{\hbar^{2}}{2 D}}
\end{array}\right) d W
$$

where $d W$ is a Wiener increment [18].

To derive a SDE for the rms position, we begin by applying Ito's formula to derive a SDE for $x_{m s} \equiv\left\langle x^{2}\right\rangle$,

$$
\begin{aligned}
& d\left(x^{2}\right)=\frac{2 p}{m} x d t \\
& \frac{d}{d t} x_{m s}=\frac{2}{m}\langle p x\rangle .
\end{aligned}
$$

Substituting $x, p$ by integrals of Eq. (6) and keeping in mind that the "functions" $d W(t)$ appearing in the expressions for $x$ and $p$ will be identical for any given stochastic realization,

$$
\frac{d}{d t} x_{m s}=\frac{\hbar^{2}}{m^{2} D}\left\langle\int_{0}^{t} d W\left(t^{\prime}\right) \int_{0}^{t} d t^{\prime \prime} \int_{0}^{t^{\prime \prime}} d W\left(t^{\prime \prime \prime}\right)\right\rangle=\frac{\hbar^{2}}{2 m^{2} D} t^{2} .
$$

Hence

$$
x_{r m s}(t)=\frac{\hbar}{m \sqrt{6 D}} t^{3 / 2}
$$

We now turn to quantify the fundamental measurement noise inherent in the present scheme. As mentioned above, the discrete measurement errors (before taking the continuous limit) are a stationary Gaussian process with variance $C \sigma / 2$. Hence, in any given time interval $B^{-1}$ the sample variance for the $N \equiv(B \tau)^{-1}$ measurements will be

$$
\begin{gathered}
\left\langle\sum \xi_{r}^{2}\right\rangle=\frac{C \sigma}{2 B \tau}, \\
\Xi_{r m s} \equiv \frac{1}{N} \xi_{r m s}=\sqrt{\frac{C \sigma B \tau}{2}} \rightarrow \sqrt{\frac{D B}{2}} .
\end{gathered}
$$

Note that $\sqrt{D}$ thus represents a measurement inaccuracy (or "sensitivity," depending on your point of view) in units such as meters $/ \sqrt{\mathrm{Hz}}$. In the following we shall regard $\Xi(t)$ as the time-domain measurement signal, so that $B$ should be interpreted as a low-pass bandwidth. It is important to understand that $D$ and $B$ are completely independent variables: $D$ parametrizes the system-meter coupling strength (a physical quantity) and $B$ the degree of smoothing applied to the measurement results (a signal-processing quantity).

We can now formulate a condition for backaction-induced diffusion to become visible against the fundamental measurement noise:

$$
\begin{aligned}
& x_{r m s}=\Xi_{r m s} \Rightarrow \frac{\hbar}{m \sqrt{6 D}} t_{*}^{3 / 2}=\sqrt{D B / 2}, \\
& t_{*}=\left[\sqrt{3 B} \frac{D m}{\hbar}\right]^{2 / 3} .
\end{aligned}
$$



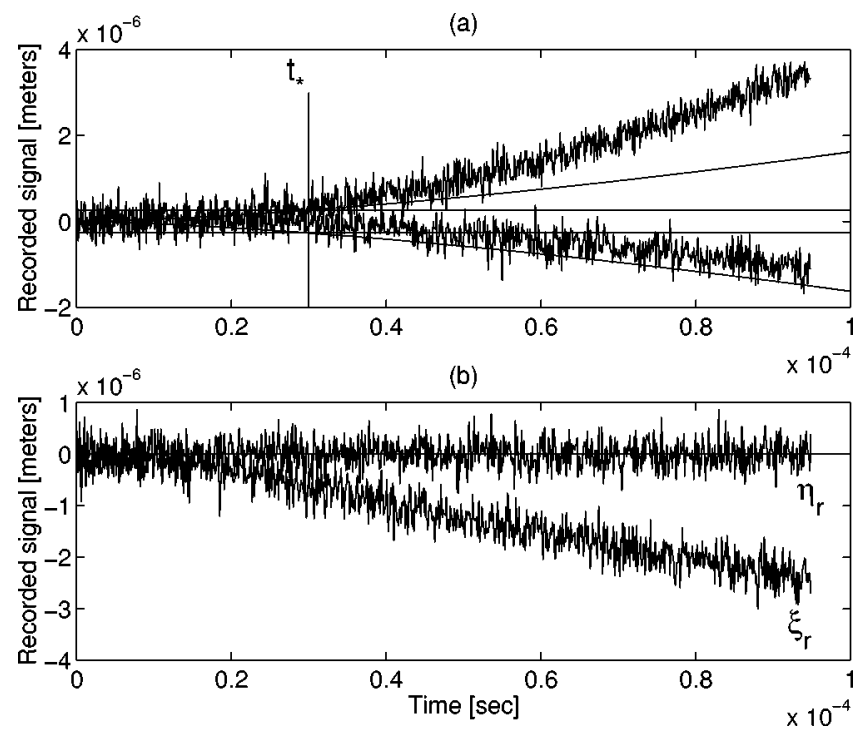

FIG. 1. (a) Horizontal lines indicate the rms signal noise $\pm \sqrt{D B / 2}$, curved lines indicate $\pm x_{r m s}(t)$, and the vertical line indicates $t_{*}$ for $D=1.42 \times 10^{-20}, B=10^{7}$, and $m=2.22 \times 10^{-25}$ (mks units). Two stochastic realizations of the measurement signal are also shown, generated by direct simulation of Eqs. (19)-(21). (b) The nonstationary component of the signal $\xi_{r}$ can be perfectly subtracted away to yield $\eta_{r} \equiv \xi_{r}-\hat{x}_{r}^{\prime}$, which is a Gaussian noise process with variance $\sigma C / 2$.

This expression should be taken to predict the overall measurement time $t_{*}$ at which backaction-diffusion should cause the rms wander $x_{r m s}$ of the observed particle (with mass $m$ ) to equal the point-to-point scatter $\Xi_{r m s}$ in continuous measurements made with measurement inaccuracy $\sqrt{D}$ and filtered to bandwidth $B$. Viewed as such, I claim that Eq. (11) represents the most sensible form for a broadband SQL for continuous observation of the position of a free mass. Note that the time scale for backaction noise to manifest itself increases with increasing signal bandwidth (measurement noise hides the backaction), increases with particle mass (this scenario is less "quantum"), and increases with measurement inaccuracy (a good measurement is needed to get to the SQL). This picture is illustrated by Fig. 1(a) (for fixed $D, B$, and $m$ ), which shows $x_{r m s}(t), \Xi_{r m s}$, and $t_{*}$ together with two stochastic realizations of $x(t)$.

In the special case where we choose to integrate the measurement results over all of a given observation interval $t$, $B \equiv 1 / t$ and we recover a single measurement scenario. We can then derive a basic figure of merit for continuous measurements: Defining $S \equiv \sqrt{D}$ as the measurement inaccuracy and keeping $t$ as the total observation time, we find that we need

$$
\frac{S}{t} \leqslant 3^{-1 / 4} \sqrt{\frac{\hbar}{m}}
$$

in order for noise in the measurement record ultimately to be dominated by real displacements of the particle due to backaction diffusion. Alternatively, we can write down something like an inference-disturbance relation

$$
\Xi_{r m s} x_{r m s} \geqslant \sqrt{\frac{D}{2 t}} \frac{\hbar}{m \sqrt{6 D}} t^{3 / 2}=\frac{\hbar t}{2 \sqrt{3} m} .
$$

This relation asserts that the product of the integrated measurement accuracy and the rms displacement of the test particle due to backaction diffusion must exceed a lower bound that increases linearly with time.

We now turn to consider the estimation of weak forces acting on the test particle, based on something like Kalman filtering of the measurement record. We begin by writing down a modified form of the stationary difference equations (still explicit)

$$
\begin{gathered}
x_{r+1}-x_{r}=p_{r} \frac{\tau}{m}+\frac{C-1}{C}\left(\xi_{r}-x_{r}^{\prime}\right), \\
p_{r+1}-p_{r}=\alpha \tau+\frac{\hbar}{\sigma \sqrt{C}}\left(\xi_{r}-x_{r}^{\prime}\right) .
\end{gathered}
$$

Here we have simply added a uniform force field $\alpha$ that acts on the particle at all times [19]. Our task will be to detect $\alpha \neq 0$.

Based on the measurement results $\xi_{r}$, we want to update recursive estimators $\hat{x}_{r}$ and $\hat{p}_{r}$ for $x_{r}$ and $p_{r}$, respectively. Assuming noiseless readout of $\xi_{r}$, the update equations read

$$
\begin{gathered}
\hat{x}_{r+1}-\hat{x}_{r}=\hat{p}_{r} \frac{\tau}{m}+\frac{C-1}{C}\left(\xi_{r}-\hat{x}_{r}^{\prime}\right), \\
\hat{p}_{r+1}-\hat{p}_{r}=\frac{\hbar}{\sigma \sqrt{C}}\left(\xi_{r}-\hat{x}_{r}^{\prime}\right) .
\end{gathered}
$$

Note that we do not include $\alpha$ in the update rules, as it is assumed to be an unknown quantity. In order to infer $\alpha$ from the recursive estimators $\hat{x}_{r}$ and $\hat{p}_{r}$, we must focus on the behavior of the quantity $\eta_{r} \equiv \xi_{r}-\hat{x}_{r}^{\prime}$. As we know the distribution for $\xi_{r}$, we can write

$$
\eta_{r}=\left(x_{r}^{\prime}-\hat{x}_{r}^{\prime}\right)+\sqrt{\frac{C \sigma}{2}} \mathcal{N}_{r}[0,1],
$$

where $\mathcal{N}_{r}[0,1]$ is a Gaussian deviate with zero mean and unit variance. Hence $\eta_{r}$ will generally be the sum of a Gaussian deviate and an uncorrelated process (the "error signal") that reflects the cumulative inaccuracy of our recursive position estimator.

Note that if there is no external force acting on the particle $(\alpha=0)$ there is no measurement inaccuracy and the recursive estimator $\hat{x}_{r}$ can be used perfectly to subtract the backaction-induced diffusion from the signal $\xi_{r}$ [see Fig. 1(b)]. Indeed, demonstrating the ability to do so would seem to be the only good way of verifying that an actual experimental broadband position measurement reaches the "quantum regime" defined by Eq. (12): Unlike the scenario of optical measurements of quadrature amplitudes, there is no way directly to compare signal and meter beams to determine the fidelity of the measurement. If there is external force, the subtraction will not be perfect and $\alpha$ will reveal itself in nonstationary behavior of $\eta_{r}$. 
Note as well that the variance of the residual noise process $\sqrt{C \sigma / 2} \mathcal{N}_{r}[0,1]$ can in principle go to zero in the limit of good measurements. At first thought this might seem to have profound implications for the detection of weak classical forces, but in fact the quantitative analysis below recovers the usual SQL for weak force detection.

Before proceeding any further, let us make the transition to the continuous limit $(\tau \rightarrow 0, \sigma \rightarrow \infty, D=$ const) [13] and collect together a complete set of SDE's for the physical quantities and statistical estimators:

$$
\begin{gathered}
D X=\frac{p}{m} d t, \quad d p=\alpha d t+\sqrt{\frac{\hbar^{2}}{2 D}} d W, \\
d \xi=x d t+\sqrt{\frac{D}{2}} d W, \\
d \hat{x}=\frac{\hat{p}}{m} d t, \quad d \hat{p}=\frac{\hbar}{D}(d \xi-\hat{x} d t) .
\end{gathered}
$$

In these terms, $d \xi / d t$ represents the broadband measurement record. Note that we could also define an auxiliary variable $e \equiv x-\hat{x}$, in terms of which

$$
\begin{gathered}
d e=\frac{1}{m}(p-\hat{p}) d t, \\
d \hat{p}=\frac{\hbar}{D} e d t+\sqrt{\frac{\hbar^{2}}{2 D}} d W .
\end{gathered}
$$

Our estimator for the external force $\alpha$ will be proportional to the time integral of $d \eta \equiv d \xi-\hat{x} d t$, so what we really need to know is the rms behavior of $e(t)$. Developing its SDE,

$$
\begin{gathered}
d e=d x-d \hat{x}, \\
\frac{d e}{d t}=\frac{1}{m}\left(\alpha t-\frac{\hbar}{D} \int_{0}^{t} e\left(t^{\prime}\right) d t^{\prime}\right), \\
\frac{d^{2} e}{d t^{2}}=\frac{1}{m}\left(\alpha-\frac{\hbar}{D} e\right) .
\end{gathered}
$$

This shows that $e(t)$ behaves like a harmonic oscillator, with mass $m$ and natural frequency $\omega_{0}=\sqrt{\hbar / m D}$, subjected to a constant external force $\alpha$. So with the initial condition $\hat{x}$ $=x, \hat{p}=p$, we expect

$$
e(t)=\frac{\alpha D}{\hbar}\left[1-\cos \left(\sqrt{\frac{\hbar}{m D}} t\right)\right] .
$$

Returning to the time integral of $d \eta$,

$$
\int_{0}^{t}\left(\frac{d \xi}{d t^{\prime}}-\hat{x}\right) d t^{\prime}=\int_{0}^{t} e\left(t^{\prime}\right) d t^{\prime}+\sqrt{\frac{D}{2}} \int_{0}^{t} d W\left(t^{\prime}\right),
$$

from which we identify the signal $\Sigma$ and rms noise $N$ as

$$
\Sigma=\frac{\alpha D}{\hbar}\left[t-\sqrt{\frac{m D}{\hbar}} \sin \left(\sqrt{\frac{\hbar}{m D}} t\right)\right], \quad N=\sqrt{\frac{D t}{2}} .
$$

Setting $\Sigma / N=1$ we obtain

$$
\alpha_{\min }=\hbar \sqrt{\frac{t}{2 D}}\left[t-\sqrt{\frac{m D}{\hbar}} \sin \left(\sqrt{\frac{\hbar}{m D}} t\right)\right]^{-1} .
$$

This expression gives a rigorous lower bound for the weakest detectable force, given particular values of $m, t$, and $D$. In order to arrive at a SQL in the traditional sense, what remains is to optimize over $D$. For fixed $m$ and $t$, it seems reasonable that the optimal choice of $D$ should be simply related to $\hbar t^{2} / m$. If we substitute $D \equiv \theta^{2} \hbar t^{2} / m$ into Eq. (28) and then (numerically) minimize over $\theta$,

$$
\alpha_{\min }=\min _{\theta} \sqrt{\frac{\hbar m}{2 t^{3}}}\left[\theta-\theta^{2} \sin (1 / \theta)\right]^{-1} \approx \pi \sqrt{\frac{\hbar m}{2 t^{3}}} .
$$

Hence we are able to recover (within a factor of $\pi$ ) the usual SQL for detecting a weak classical force via repeated position measurements on a free mass [4].

Looking back at Eqs. (19)-(21), note that we are allowed to substitute an arbitrarily time-varying external force $\alpha(t)$ without violating the conditions of the derivation (Gaussian propagation). Hence one arrives at the obvious extension of Eq. (24):

$$
\frac{d^{2} e}{d t^{2}}=\frac{1}{m}\left(\alpha(t)-\frac{\hbar}{D} e\right)
$$

The time development of the Kalman estimator $e(t)$ therefore corresponds to the response of a simple harmonic oscillator (SHO) to the applied force $\alpha(t)$. All the quantum measurement phenomenology seems to be distilled into the single fact that the stiffness (resonance frequency) of this SHO scales inversely with the inaccuracy of the broadband position measurements. Computing this response gives the exact wave form $e(t)$ that would be induced by any expected signal. Knowing this, one could easily design a matched filter [20] for optimal detection against the Gaussian white noise $\sqrt{D t / 2}$.

Considering the simplest case $\alpha(t)=\alpha \cos (f t)$, we could guess that an interesting response might be obtained by choosing $D$ such that $f=\sqrt{\hbar / m D}$. Under these conditions we can guess that $e(t)=A(t) \sin (f t)$, so that

$$
\begin{aligned}
\frac{d^{2} e}{d t^{2}} & =\left(\frac{\alpha}{m} \cos (f t)-f^{2} A(t) \sin (f t)\right) \\
& =2 f \dot{A} \cos (f t)+\left(\ddot{A}-f^{2} A\right) \sin (f t) .
\end{aligned}
$$

Therefore, $A(t)=\alpha t(2 m f)^{-1}$ and

$$
e(t)=\frac{\alpha t}{2 m f} \sin (f t)
$$




$$
f=\sqrt{\frac{\hbar}{m D}}
$$

The matched filter for this will be simply $\sin (f t)$ and

$$
\begin{aligned}
\int_{0}^{t} d t^{\prime} e\left(t^{\prime}\right) \sin \left(f t^{\prime}\right)= & \frac{\alpha}{2 m f^{3}} \int_{0}^{t} d\left(f t^{\prime}\right)\left(f t^{\prime}\right) \sin ^{2}\left(f t^{\prime}\right) \\
= & \frac{\alpha}{2 m f^{3}}\left[\frac{1}{4} \sin ^{2}(f t)\right. \\
& \left.-\frac{f t}{2} \sin (f t) \cos (f t)+\frac{1}{4} f^{2} t^{2}\right]
\end{aligned}
$$

The interesting regime would seem to be $f t \gg 1$, where the integrated error signal goes like $t^{2}$ (as opposed to $t$ in the case of constant $\alpha$ ). Setting $\Sigma$ to the expression above and $N=\frac{1}{2} \sqrt{D t / 2}$, we have

$$
\begin{aligned}
\alpha_{\min } & \sim 4 m f t^{-2} \sqrt{D t / 2} \\
& =4 \sqrt{\frac{\hbar m}{2 t^{3}}} .
\end{aligned}
$$

One sees that the use of Eq. (30) together with some intuition about the behavior of forced harmonic oscillators provides a convenient way of thinking about classical force detection in the quantum regime of strong measurement backaction.

The author would like to thank G. J. Milburn, J. W. Hartman, and Y. Levin for discussions and to acknowledge support from the National Science Foundation under Grants Nos. PHY94-07194 and PHY97-22674.
[1] H. P. Yuen, Phys. Rev. Lett. 51, 719 (1983).

[2] C. M. Caves, Phys. Rev. Lett. 54, 2465 (1985).

[3] M. Ozawa, Phys. Rev. Lett. 60, 385 (1988).

[4] V. B. Braginsky and F. Ya. Khalili, Quantum Measurement (Cambridge University Press, Cambridge, 1992).

[5] M. T. Jaekel and S. Reynaud, Europhys. Lett. 13, 301 (1990).

[6] V. B. Braginsky and Yu. I. Vorontsov, Ups. Fiz. Nauk 114, 41 (1974) [Sov. Phys. Usp. 17, 644 (1975)].

[7] C. M. Caves, K. S. Thorne, R. W. P. Drever, V. D. Sandberg, and M. Zimmermann, Rev. Mod. Phys. 52, 341 (1980).

[8] C. M. Caves and G. J. Milburn, Phys. Rev. A 36, 5543 (1987).

[9] G. J. Milburn, K. Jacobs, and D. F. Walls, Phys. Rev. A 50, 5256 (1994).

[10] A. C. Doherty, A. S. Parkins, S. M. Tan, and D. F. Walls, Phys. Rev. A (to be published).
[11] H. Mabuchi, J. Ye, and H. J. Kimble (unpublished).

[12] O. L. R. Jacobs, Introduction to Control Theory (Oxford University Press, Oxford, 1993).

[13] G. J. Milburn, Quantum Semiclassic. Opt. 8, 269 (1996).

[14] H. Mabuchi, Quantum Semiclassic. Opt. 8, 1103 (1996).

[15] H. M. Wiseman, Phys. Rev. Lett. 75, 4587 (1995).

[16] H. M. Wiseman, Phys. Rev. A 49, 2133 (1994).

[17] V. P. Belavkin and P. Staszewski, Phys. Rev. A 45, 1347 (1992).

[18] C. W. Gardiner, Handbook of Stochastic Processes (Springer, Berlin, 1990).

[19] Equations (14) and (15) can be derived by direct solution of the Schrödinger equation with a Gaussian ansatz.

[20] C. W. Helstrom, Elements of Signal Detection and Estimation (PTR Prentice-Hall, Englewood Cliffs, NJ, 1995). 